\title{
Improving the general health of people with learning disabilities
}

\author{
Mike Kerr
}

\begin{abstract}
People with learning disabilities suffer an increase in common morbidity, communication difficulties, a high prevalence of serious conditions such as epilepsy, and specific patterns of health needs associated with the aetiology of their disability. Unfortunately, this combination of need is mirrored by a consistent picture of poor health promotion uptake, inadequate care for serious morbidity, unrecognised health need and poor access to health care. Consequently, there is a great disparity between the health of learning-disabled people and that of the general population. Psychiatrists can address this disparity in clinical practice by focusing on these patients' mental health, epilepsy management and the impact of behaviour on health. They can also influence health planning and service development.
\end{abstract}

\section{Inequalities in health status}

The achievement of good health is an appropriate goal for all, including people with learning disabilities. This brings particular challenges, as individuals with learning disabilities are a heterogeneous population with varying needs, who receive a similarly complex array of health care provision. Furthermore, the impact of societal and environmental factors on health status is arguably greater in this group. The imperative to remove the great disparity between the health of learningdisabled people and that of the general population is strong, because improved health is likely to improve quality of life, both of individuals and their families. In this article I make the premise that the general health of learning-disabled people can be improved by addressing those areas in which disparities in health and in health care provision are evident. These include:

- a difference in health because of:

- increased mortality

- increased morbidity

- increase in negative determinants of health such as poverty;

- a difference in health care because of:

- unequal access to services

- inequality of services.

To focus on how health improvements can be made, I consider here the following five areas: the disparity in health; health needs; barriers to health care; health provision in primary care; addressing the disparity.

\section{The disparity in health}

If an appropriate aim for any improvement in health for this population is to reach at least the same level of health and access to health care as that enjoyed by the general population, then it is necessary to address the issues of disparity in health. In the past, the concept of health disparity has mainly been applied to minority ethnic or other special groups (such as those facing social deprivation) to show inequality in health status and health access between such groups and the general population. More recently, interest has focused on the disparity for people with learning disabilities (Surgeon General, 2002).

The concept is important and can add a focus to the planning of services. It does, however, present some difficulties, particularly when trying to allow for the impact of the aetiology of the individual's disability on any apparent disparity. An example of this would be the reduced life expectancy seen in profound learning disability. To address this problem, comparison within groups who share the same disability may be needed. This is not to say that some aspects of mortality in the different groups are not preventable. For example, continued advances in accident prevention and in the treatment of swallowing abnormalities and epilepsy may

Mike Kerr is Professor of Learning Disabilities and an honorary consultant in neuropsychiatry at the Welsh Centre for Learning Disabilities (Department of Psychological Medicine, University of Wales College of Medicine, Meridian Court, North Road, Cardiff CF14 3BG, UK. E-mail: kerrmp@cf.ac.uk). 
Table 1 Health disparity in people with learning disabilities

Area of disparity

Increased mortality

Increased morbidity

Increase in negative

determinants of health

Access to services

Quality of services
Example in learning disability

Lower life expectancy

High levels of epilepsy, sensory impairment and behavioural disorder

High levels of obesity and underweight; low employment; fewer social connections and meaningful relationships

Low rates of uptake of health promotion

High prescription rate of antipsychotic medication with no evidence of psychosis; high rates of unrecognised disease identified on health screening well reduce disparity in some groups. Table 1 highlights key disparity issues.

\section{Health needs}

Box 1 summarises the main findings of the 1995 Welsh Health Survey (Welsh Office, 1996), a large questionnaire-based survey that provides a baseline for the community health status of individuals with learning disabilities.

People with learning disabilities have both general and special health needs. General health needs include the treatment of acute and chronic illness, health promotion and appropriate hospital or other referral. These should be met within a primary health care setting. Specialist health needs can be divided into common morbidities that occur at a greater frequency and patterns of health need associated with specific medical conditions.

These conditions are not unique to people with learning disabilities, but their high prevalence in

\section{Box 1 The Welsh Health Survey: summary of findings (Welsh Office, 1996)}

The following results are specific to people with learning disabilities:

- They had a higher rate of psychiatric illness $(32.2 \%)$ than the general population $(11.2 \%)$

- They had more illness than the general population: only $6 \%$ reported no illness, compared with $37 \%$ of the general population

- The prevalence of epilepsy was $22.1 \%$

- Eyesight was poor in $19.1 \%$, compared with $8 \%$ of the general population

- The family doctor was the most frequently contacted professional in the previous year $(79.5 \%)$

- Only 1 in 10 individuals had a healthy diet, and 1 in 3 had an unhealthy diet

- $28.8 \%$ were overweight and $23.6 \%$ were obese this population means that they are an important part of any assessment. A reasonable consensus can be made that sensory deficit, epilepsy, and behavioural and psychological disturbance in particular are more common in learning-disabled people.

\section{Sensory deficit: vision and hearing}

High levels of sensory impairment are common in people with learning disabilities. An institutional survey of vision (McCulloch et al, 1996) showed that $12 \%$ of mildly disabled people, more than $40 \%$ of severely disabled and $100 \%$ of profoundly disabled people had poor visual acuity. The prevalence of ocular health problems ranged from $25 \%$ in the mildly disabled group to $60 \%$ in the profoundly disabled group. A study of severely disabled children and adolescents in another residential institution reflected this high prevalence (Kwok et al, 1996). Here $25 \%$ had severe visual impairment and $24 \%$ refractive errors. Evenhuis et al (1997) identified hearing loss in $25-42 \%$ of community samples of people with learning disabilities.

\section{Epilepsy}

The association between severity of learning disability and the prevalence of epilepsy is reflected in the wide variation of prevalence figures in the literature. Institutionalised populations are more likely to contain those with severe disability. This is reflected in Mariani et al's (1993) finding of a $32 \%$ prevalence of epilepsy in an institutional study, whereas Chadwick (1994) estimated that the prevalence in the general population was between $0.4 \%$ and $1 \%$. A more recent community study of epilepsy in learning-disabled people showed a prevalence of $16.1 \%$ (Morgan et al, 2003).

\section{Syndrome-specific conditions}

Certain syndromes that cause learning disability are particularly associated with an increased risk of specific morbidity. Down's syndrome, for example, 
is associated with increased risks of cardiovascular disease, respiratory disease, eye disorders, leukaemia. Alzheimer's disease and hypothyroidism. People with fragile- $X$ syndrome have increased connective tissue disease, leading to joint laxity and cardiac abnormalities. Major disorders in the control of satiety have been recognised in Prader-Willi syndrome, with an associated risk of obesity-related pathology.

\section{Mortality and learning disability}

There is a higher mortality rate (seen in both standardised mortality ratios and reduced life expectancy) in people with learning disabilities, compared with the general population (Morgan et al, 2001). However, this must be balanced against a trend towards longer life expectancy in this population (Richards \& Siddiqui, 1980). Predictors of mortality include the severity of the learning disability, reduced mobility, feeding difficulties and Down's syndrome (Strauss et al, 1998; Van Allen et al, 1999). Additionally, it appears that learning-disabled people with epilepsy have increased mortality and are at greater risk of sudden unexplained death in epilepsy (Forssman \& Aksesson, 1970; Leestma et al, 1989).

\section{Barriers to health care}

A potential cause of the disparity between the health of people with learning disabilities and that of the general population is the concept of barriers to care. Several such barriers have been suggested (Table 2), and it is likely that all of these, often interacting, play a role in disparity. Certainly, the clinician will need to recognise the potential existence of these barriers and minimise them where possible.

\section{Mobility}

In a North American study of 614 physicians it was reported that $12 \%$ worked in offices inaccessible to patients with mobility problems (Minihan et al, 1993).

\section{Sensory impairment}

Sensory impairment may reduce the ability of patients to attend appointments unaccompanied. It can also increase their distress during consultations and physical examinations, as communication and comprehension are reduced.

\section{Behavioural problems}

In the USA, Minihan \& Dean (1990) found that 20\% of learning-disabled patients could be examined or treated only after measures such as pre-medication or pre-visits for desensitisation had been taken. Only one in five primary care physicians reported that they felt well prepared to handle a patient who refused to cooperate with an examination or treatment (Minihan et al, 1993).

Behavioural problems may hinder diagnosis of conditions if investigations such as electroencephalograms are needed. In rare cases of epilepsy, doubt may exist as to whether a behavioural outburst does in fact represent a seizure (IASSID Guidelines Group, 2001).

\section{Communication}

The reliance on carers to communicate health needs has been cited as a major barrier to health care. Wilson \& Haire (1990) discovered health problems that had been over looked in instances when carers believed the person in their care to be in good health. In the same study, the authors found that carers failed to predict sensory impairment in 50\% of patients who had difficulties in hearing or seeing.

\section{Knowledge, attitudes and access to specialist services}

Lennox et al (1997) found that Australian primary care physicians listed lack of knowledge of conditions and illnesses specific to learning disability as among the top five barriers to care.

\section{Table 2 Barriers to health care}

$\begin{array}{ll}\text { Domain } & \text { Effect } \\ \text { Mobility } & \text { Difficulty accessing health services } \\ \text { Sensory impairment } & \text { Reduced communication and comprehension of health processes } \\ \text { Behaviour problems } & \text { Difficulty in examination and investigation of disease } \\ \text { Communication } & \begin{array}{l}\text { Reduced comprehension of health processes; difficulty in presenting disease } \\ \text { owing to reduced communicative skills; poor communicative skills of health } \\ \text { professionals }\end{array} \\ \text { Knowledge, attitudes and } & \text { Poor professional knowledge; attitudinal barriers to accessing care } \\ \text { accessibility of specialist services } & \end{array}$


Also in Australia, Beange et al (1995) found that $74 \%$ of 202 individuals with learning disabilities had conditions for which specialist care was needed. This care had, however, not always been received, and half of the identified conditions were found to be inadequately managed.

\section{Does primary care meet the health needs of learning-disabled people?}

The three key papers in this area give consistent findings (Howells, 1986; Beange \& Bauman, 1990, 1991; Wilson \& Haire, 1990). These studies (from Wales, Australia and England respectively) suggest that examination of any community-based population of people with learning disabilities receiving primary care will consistently uncover three serious problems:

- untreated, yet treatable, medical conditions

- untreated specific health issues related to the individual's disability

- a lack of uptake of generic health promotion such as blood-pressure screening.

The lack of control data in these initial studies reduces their impact, as it is difficult to reflect on which aspects were specifically disability-related: the surveys might well have reflected generic difficulties in the delivery of primary health care.

Subsequent studies (Lagan et al, 1993; Beange et al, 1995; Whitefield et al, 1996) used controlled comparison data. This has allowed for comparative information on health status, health promotion activity and service contact.

Beange et al (1995) in Australia found significant greater levels of obesity, vision and hearing impairment, hypersomnia, endocrine disease, skin disease and psychiatric disorders in adults with learning disabilities. Additionally, non-Down's syndrome women had increased hypertension and showed a lack of sufficient exercise. The control population used were 'population norms' from the National Heart Foundation survey of the general population. Whitefield et al (1996) compared a random sample of learning-disabled people taken from the patient register of a primary health care team in an English city with age- and gendermatched controls from the same register. The study found a significant increase in the use of antipsychotic and anticonvulsant medication and an increase in consultations for neurological and skin conditions in the people with learning disabilities. The control population was significantly more likely to receive regular blood-pressure monitoring.
These studies indicate a lack of uptake of health promotion in people with learning disabilities. This is unlikely to be due to lower levels of contact with primary health care teams. Research in the UK, USA and Australia suggests that people with learning disabilities visit their general practitioners at least as regularly as do the general population (Jacobson et al, 1989; Beange et al, 1995; Kerr et al, 1996a; Welsh Office, 1996). Furthermore, they have been shown to use specialist services and to be hospitalised more frequently than the general population (Beange et al, 1995; Kerr et al, 1996b).

\section{Addressing the disparity}

To decrease the disparity between the health of people with learning disabilities and that of the general population, two separate key areas need to be discussed: first, primary care and second, specialist psychiatric care.

\section{Primary care}

Primary care provision is central to improving the health of people with learning disabilities yet, as we have seen, it is currently inadequate.

\section{Assessment}

Assessment should enable a physician to recognise the potential morbidities and likely problems that might be seen in clinical practice. As individuals with learning disabilities often present vague symptoms compounded by communication difficulties, clinicians should follow a structured assessment comprising:

- assessment of the initial complaint

- recognition and assessment of comorbidity

- recognition of health promotion status.

Unfortunately, attempts in the UK to carry out this more structured assessment in normal primary care have been shown to be unsuccessful (Jones \& Kerr, 1997). Therefore a change in the way consultations are carried out is necessary that will require primary care teams both to alter practice organisation and to acquire new clinical competencies. There is no agreed format for how such changes can be made. Whether practices alone will take responsibility or a lead will be provided by learning disability partnership boards (Department of Health, 2001) is not known. It seems likely that practices will need considerable support, most probably to utilise skill held within specialist learning disability services. Leadership and guidance will be needed, as people with learning disabilities are not included among the target populations listed in the new general 
practioners' contract. However, some of the recommendations to enhance services that are given below may address this issue.

\section{Practice organisation}

In England, health care policy is directed by the Government White Paper Valuing People (Department of Health, 2001), which identifies the improved health care of people with learning disabilities as a key outcome. Box 2 lists the main items in this document relating to the health of learning-disabled people. The White Paper is a little less clear on how these aims will be achieved, but likely changes needed in primary care will include a practice register, a recall system and improved communication with other services.

Practice registers Practices will need to be able to identify all individuals with learning disabilities on their lists, and this requires a comprehensive practice register. Identification from existing lists can be performed using keyword searches for common conditions such as Down's syndrome, and also by asking practice staff. Once the register is established, the recording of names should be proactive.

Recall and audit Recall is necessary to assess uptake of health promotion. The presence of Health Action Plans may be an appropriate audit topic. It is likely that recall is best served by a 'one-stop shop' annual health check such as the Cardiff Health Check (Fraser et al, 1998: pp. 293-299), which covers health promotion, physical examination and morbidity specific to learning-disabled people, such as epilepsy and behavioural disturbance.

Contact with other services Practices will need to ensure that the number of contacts with and patterns of referral to disability services and health facilitators are clearly established and recorded.

Box 2 Key points in Valuing People (Department of Health, 2001)

Government policy states that all learningdisabled people in England:

- have right of access to mainstream health services

- should each have an identified health facilitator by 2003

- should each be registered with a general practitioner by 2004

- should each have their own Health Action Plan by 2005

\section{Clinical competencies}

Primary care teams will need skills specific to the care of people with learning disabilities: health assessment, health promotion, communication and ethics, assessment of need and referral patterns for complex pathology, and specific skills such as epilepsy management.

\section{Specialist psychiatric care}

Specialist psychiatric care in the context of a multidisciplinary team primarily provides specific psychiatric assessment and treatment. Its broader function as an organiser of and conduit to general health care services is, though less well defined, equally important. In both of these areas, key clinical competencies are required, particularly when medical and psychiatric needs combine, as for example in issues relating to the treatment of epilepsy in people with learning disabilities. Table 3 identifies these functions and necessary competencies. Of particular importance in learning disability is the presentation of physical disease through psychiatric symptoms. This is commonly seen when pain presents as agitation. The chronic pain and anorexia associated with reflux oesophagitis and gastric ulcer disease mimic depressive symptoms. There should be particular diagnostic caution with learning-disabled patients whose 'depression' is associated with sleep disturbance and agitation.

\section{Conclusions}

Considerable evidence points to a disparity between the health of people with learning disabilities and that of the general population. Psychiatric practice can be central to ameliorating this situation. Psychiatric interventions for common mental illnesses and complex behavioural problems in learning disability can have a direct impact on health status. Perhaps the most important function of psychiatric practitioners in this area is to act as catalysts and drivers of general health improvement by facilitating patients' access to the appropriate pathways to care.

\section{References}

Beange, H. \& Bauman, A. (1990) Caring for the developmentally disabled in the community. Australian Family Physician, 19, 1558-1563.

Beange, H. \& Bauman, A. (1991) Health care for the developmentally disabled: is it necessary? In Key Issues in Mental Retardation Research (ed. W. I. Fraser). London: Routledge. 
Table 3 Functions of psychiatric practice in delivering health care to people with learning disabilities

Function

Psychiatric illness

Treatment and assessment

of all psychiatric illness

Treatment and assessment

of challenging behaviour

Interface between epilepsy and psychiatric illness

Diagnostic assessment

Treatment of epilepsy

Treatment of mental illness

coexistent with epilepsy

Health care organisation

Recognition of psychiatric

symptoms as presentation

of physical morbidity

Appropriate referral to health

care specialty through

general practitioner
Health improvement

Reduced morbidity in

individuals and carers

Reduced seizure-related morbidity and mortality Reduce inaccurate diagnosis Identified and treated comorbid mental illness

Reduction in inappropriate psychiatric diagnosis and treatment Decrease in physical ill health
Clinical competencies

Knowledge of presentation of mental illness in those with communication deficits Assessment of behaviour disorder Knowledge of pharmacological, behavioural and psychotherapeutic treatments

Knowledge of seizure types and their presentation as behaviour disorder or mental illness

Knowledge of epilepsy treatment Knowledge of diagnostic standards for epilepsy

Knowledge of behavioural assessment

Knowledge of patterns of unrecognised physical ill health in people with a learning disability

Knowledge of appropriate referral pathways
Beange, H., McElduff, A. \& Baker, W. (1995) Medical disorders of adults with mental retardation. A population study. American Journal of Mental Retardation, 99, 595604.

Chadwick, D. (1994) New options for treatment of epilepsy: topiramate in perspective. Epilepsia, 38, 20-44.

Department of Health (2001) Valuing People: A New Strategy for Learning Disability for the 21st Century. London: Stationery Office.

Evenhuis, H. M., Mul, M., Lemaire, E. K. G., et al (1997) Diagnosis of sensory impairment in people with intellectual disability in general practice. Journal of Intellectual Disability Research, 41, 422-429.

Forssman, H. \& Aksesson, H. O. (1970) Mortality of the mentally deficient. A study of 12,903 institutionalised subjects. Journal of Mental Deficiency Research, 14, 276294.

Fraser, W., Sines, D. \& Kerr, M. (eds) (1998) The Care of People with Intellectual Disabilities (9th edn). Oxford: ButterworthHeinemann.

Howells, G. (1986) Are the medical needs of mentally handicapped adults being met? Journal of the Royal College of General Practitioners, 36, 449-453.

Jacobson, J. W., Janicki, M. P. \& Ackerman, L. J. (1989) Health care service usage by older persons with developmental disabilities living in community settings. Adult Residential Care Journal, 3, 181-191.

Jones, R. G. \& Kerr, M. P. (1997) A randomized control trial of an opportunistic health screening tool in primary care for people with intellectual disability. Journal of Intellectual Disability Research, 41, 409-415.

IASSID Guidelines Group (2001) IASSID clinical guidelines for the management of epilepsy in adults with an intellectual disability. Seizure, 10, 401-409.

Kerr, M., Richards, D. \& Glover G. (1996a) Primary care for people with an intellectual disability - a group practice study. Journal of Applied Research in Intellectual Disabilities, 9, 347-352.

Kerr, M., Thapar, A. \& Dunstan, F. (1996b) Attitudes of general practitioners to people with a learning disability. British Journal of General Practice, 46, 92-94.

Kwok, S. K, Ho, P. C. P., Chan, A. K. H., et al (1996) Ocular defects in children and adolescents with severe mental deficiency. Journal of Intellectual Disability Research, 40, 330335.

Lagan, J., Russell, O. \& Whitefield, M. (1993) Community Care and the General Practitioner: Primary Health Care for People with Learning Disabilities. Bristol: Norah Fry Research Centre.

Leestma, J. E., Walczak, T., Hughes, J. R., et al (1989) A prospective study of sudden unexpected death in epilepsy. Annals of Neurology, 26, 195-203.

Lennox, N. G., Diggens, J. N. \& Ugoni, A. M. (1997) The general practice care of people with intellectual disability: barriers and solutions. Journal of Intellectual Disability Research, 41, 380-390.

Mariani, E., Ferini-Strambi, L., Sala, M., et al (1993) Epilepsy in institutionalized patients with encephalopathy. Clinical aspects and nosological considerations. American Journal on Mental Retardation, 98, 27-33.

McCulloch, D. L., Sludden, P. A., McKeon, K., et al (1996) Vision care requirements among intellectually disabled adults. Journal of Intellectual Disability Research, 40, 140150.

Minihan, P. M. \& Dean, D. H. (1990) Meeting the needs for health services of persons with mental retardation living in the community. American Journal of Public Health, 80, 1043-1048.

Minihan, P. M., Dean, D. H. \& Lyons, C. M. (1993) Managing the care of patients with mental retardation: a survey of physicians. Mental Retardation, 31, 239-246.

Morgan, C. Ll., Scheepers, M. I. A. \& Kerr, M. P. (2001) Mortality in patients with intellectual disability and epilepsy. Current Opinion in Psychiatry, 14, 471-475.

Morgan, C. Ll., Baxter, H. \& Kerr, M. P. (2003) Prevalence of epilepsy and associated health service utilisation and mortality among patients with intellectual disability. American Journal on Mental Retardation, 108, 293-300.

Richards, B. W. \& Siddiqui, A. Q. (1980) Age and mortality trends in residents of an institution for the mentally handicapped. Journal of Mental Deficiency Research, 24, 99-105.

Strauss, D., Anderson, T. W., Shavelle, R., et al (1998) Causes of deaths of persons with developmental disabilities. Comparison of institutional and community residents. Mental Retardation, 36, 386-391. 
Surgeon General (2002) Closing the Gap: A National Blueprint to Improve the Health of Persons with Mental Retardation. Washington, DC: US Department of Health and Human Services.

Van Allen, M. I., Fung, J. \& Jurenka, S. B. (1999) Health care concerns and guidelines for adults with Down syndrome. American Journal of Medical Genetics, 89, 100-110.

Welsh Office (1996) Welsh Health Survey 1995. Cardiff: Welsh Office.

Whitefield, M. L., Lagan, J. \& Russell, O. (1996) Assessing general practitioners' care of adult patients with learning disability: case control study. Quality in Health Care, 5, $31-35$.

Wilson, D. N. \& Haire, A. (1990) Health care screening for people with mental handicap living in the community. BMI, 301, 1379-1381.

\section{Multiple choice questions}

1 As regards people with learning disabilities:

a they have a $50 \%$ prevalence of epilepsy

b have rates of psychiatric illness of at least $30 \%$

c $80 \%$ have poor vision

d more than $50 \%$ are clinically overweight

e $40 \%$ have a healthy diet.

2 Reduced life expectancy in people with learning disabilities has been shown to be associated with:

a psychiatric illness

b mobility problems

c feeding difficulties d epilepsy

e behaviour abnormality.

3 The following have been identified as barriers to receiving health care:

a behaviour abnormality

b gender

c mobility

d the treating psychiatrist's knowledge

e communication.

4 Problems identified in the primary care of people with learning disabilities include:

a untreated medical conditions

b greatly reduced access to appointments

c lack of uptake of health promotion

d patients often being removed from general practitioners' lists

e specialist health issues not being addressed.

5 Actions identified to improve health care in Valuing People include:

a annual health checks in primary care

$\mathrm{b}$ health facilitators

c more community learning disability nurses

d all patients are to be registered with a general practitioner

e Health Action Plans for each patient.

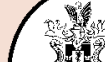

